\title{
CONSTRAINTS ON STELLAR EVOLUTION THEORY FROM PRECISE ECLIPSING BINARY DATA
}

J.Andersen, J.V.Clausen, H.E.Jørgensen and B.Nordström Copenhagen University Observatory, Denmark

Previous attempts at a detailed confrontation of eclipsing binary data with theoretical models of main-sequence evolution were faced with the choice between data of inhomogeneous (mostly low) quality for many systems (Křiž, 1969; Lacy, 1979) or accurate values of mass, radius, and temperature (or luminosity) for very few systems only (Popper et al., 1970). In addition, more detailed and homogeneous stellar structure calculations for several compositions were needed. Since 1972, a coordinated photometric and spectroscopic programme at our institute contributes to building a sufficient observational basis for such a test. Among published standard models for the range $1-10 \mathrm{M}_{\odot}$, Hejlesen's (1980) are the most extensive, agree well with other standard models, and are presented in a format suitable for comparison with binary data. Here we can only outline a few salient new results from this study.

Experience in comparing with theoretical models shows that for useful discrimination between models of different chemical composition, mixing length, etc., a precision in the observed values of $\log M$, log $R$, and $\log$ Te of about 0.01 (0.03 in $\log g$ ) or better is required. Fig.l displays the existing fundamental data ( $M$ and $R$ ) in this class (Popper, 1980 and later; later published and unpublished data by our group), and shows main sequence boundaries and isochrones for Hejlesen's models for $(X, Z)=(0.70,0.02)$. Although parts of it are still sparsely populated, this diagram shows very good agreement with the observations (same age within a system) for $Z$ close to 0.02 in a wide range of $Y$. Better coverage of strategic parts of this diagram should permit a direct test of such effects as mixing, overshooting, etc., but in this mass range, standard models apparently predict correctly the evolution in radius, as shown by the systems with components of appreciably different mass.

However, when also the observed temperatures are compared with the model predictions, we obtain the unexpected result that the systems do not fit one single composition, but fall into two distinct groups: the $B-A l$ stars fit $(X, Z)=(0.70,0.02)$ and the later spectral 


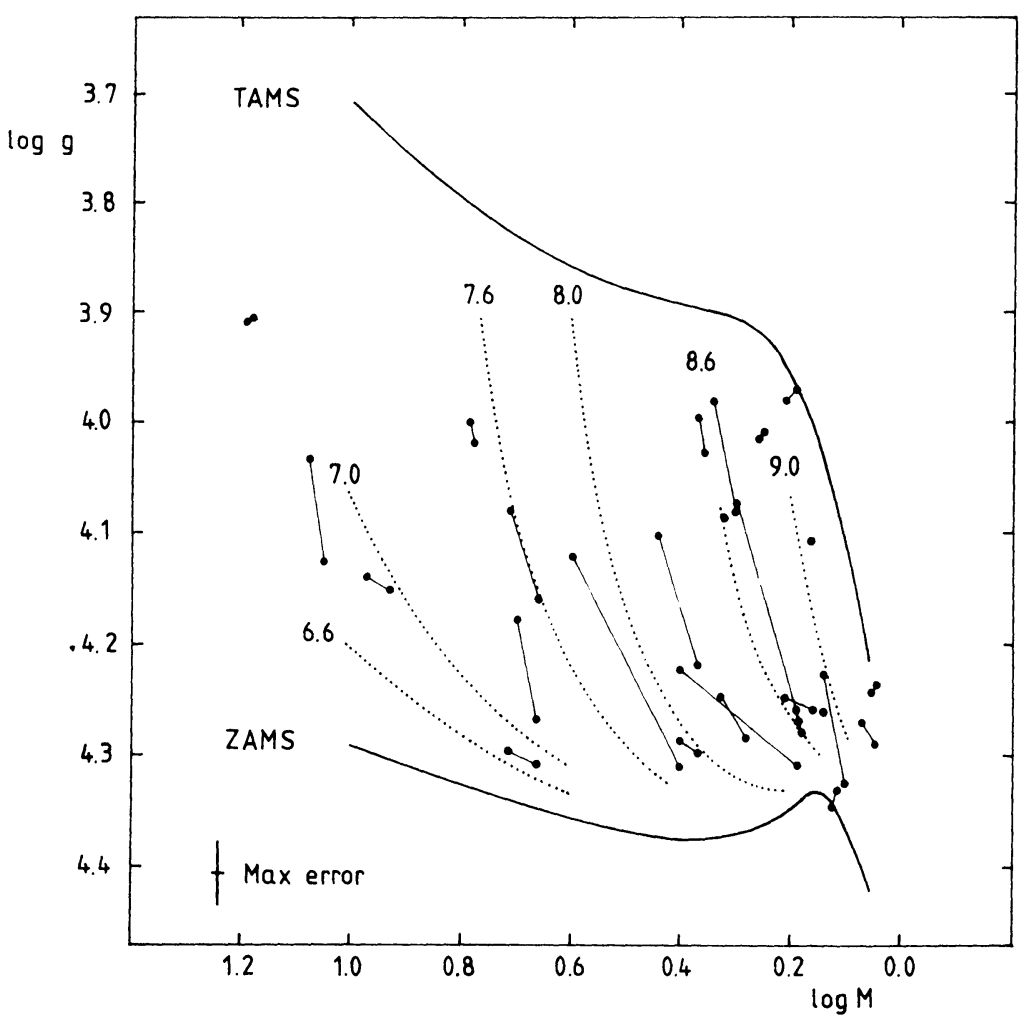

Fiq.l. loq M - loq a diaqram comparina the best available data with main-sequence boundaries and isochrones (with log age) by Hejlesen for $(X, Z)=(0.70,0.02)$.

types $(X, Z)=(0.80,0.02)$. This effect is well seen in the $\log \mathrm{Te}$ log $g$ diagram, and Fig.2 shows the region of it where the stars separate into the two groups. The systems are plotted on the theoretical tracks according to their observed $\log M$ and $\log g$ values, and the error bars in $\log$ Te indicate the range in model temperatures corresponding to the observed mass limits. Arrows point to the observed log $T e$ when the difference from the model prediction exceeds 0.01 . To the left in the diagram (and higher masses) there is good agreement, to the right (and lower masses) observed log Te's are lower, by about 0.05 . Choosing models of $(X, Z)=(0.80,0.02)$ [or $(X, Z)=(0.70,0.04)$ but those are excluded by other data] would reverse the situation. However, the two groups overlap in such single parameters as relative radii, orbital eccentricity, mass, temperature, gravity, surface rotation, and age, which makes it difficult to ascribe the effect to systematic errors in the models or temperature calibrations - or indeed to abundance differences. A third parameter (differential rotation, magnetic fields?) seems to be operating.

Forthcoming papers will treat these comparisons in more detail. 


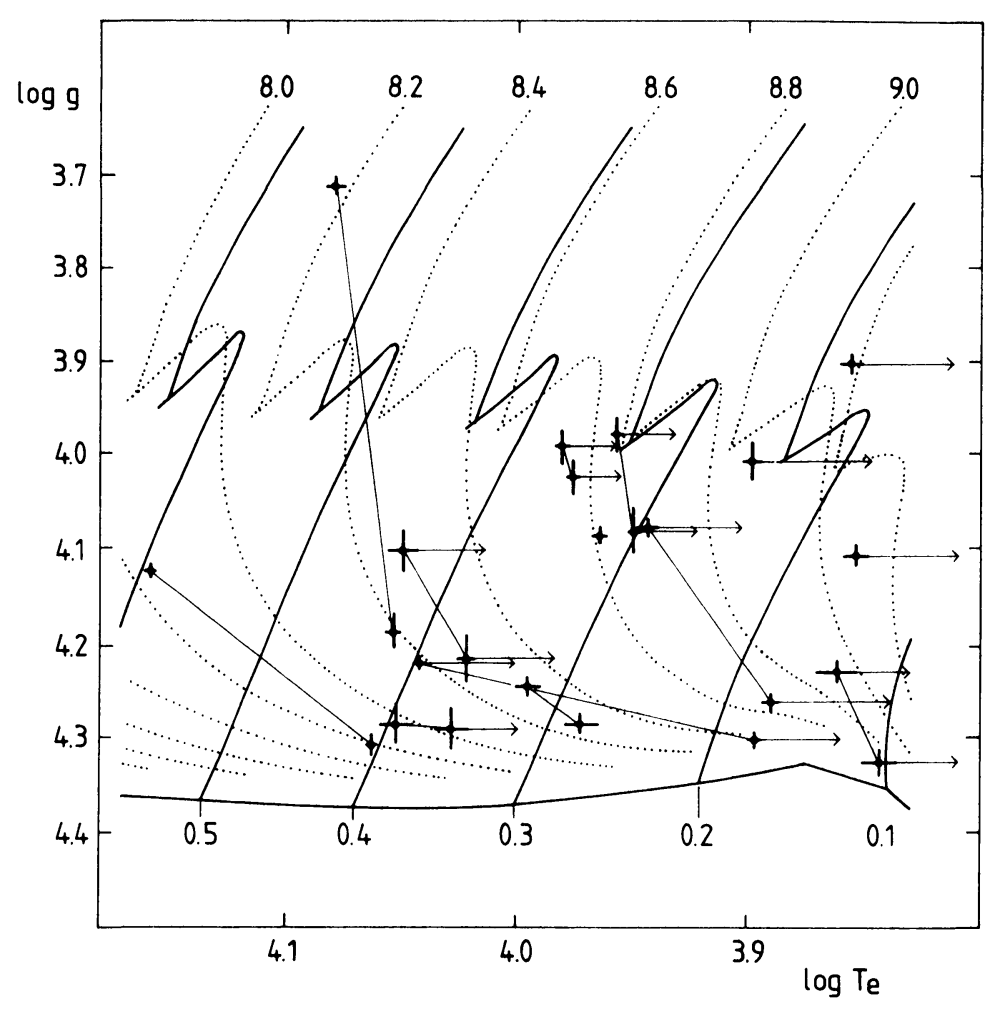

Fig.2. Evolutionary tracks (full lines, with $\log M$ ), isochrones (dotted lines, with $\log$ age), and observed systems in the log Te - log $g$ diagram ( see text); $(X, Z)=(0.70,0.02)$.

This work is supported by ESO observing time, by the Danish Natural Science Research Council and the Danish Board for Astronomical Research. Several colleagues have contributed to individual systems and/or to discussions of the results.

\section{REFERENCES}

Hejlesen, P.M.: 1980, Astron. Astrophys. Suppl. Ser. 39, 347 Kř̉z, S.: 1969, Bull. Ast. Inst. Csl. 20, 202 Lacy, C.H.: 1979, Ap. J. 228, 817 Popper, D.M.: 1980, Ann.Rev. Astron. Astrophys. 18, 115

Popper, D.M., Jørgensen, H.E., Morton, D.C., Leckrone, D.S.: 1970, Ap. J. 161, L 57 . 


\section{DISCUSSION}

Kudritzki: How did you obtain the effective temperature of your objects?

Clausen: For systems analysed in the Copenhagen project we have uvby light curves. The standard 4-colour indices for each component can therefore be calculated and the various available $\mathrm{T}$ eff calibrations used (and compared). See our papers in Astron. Astrophys. For references. For the other systems included the $T_{\text {eff }}$ values were taken from Popper's (1980) review article and therefore fased on the calibration given there. 